\title{
Integrated silicon nitride electro-optic modulators with atomic layer deposited overlays
}

\author{
Artur Hermans, ${ }^{1,2, *}$ (1) Michiel Van Daele, ${ }^{3}$ Jolien Dendooven, ${ }^{3}$ Stéphane Clemmen, ${ }^{1,2,4}$ (1) \\ Christophe Detavernier, ${ }^{3}$ and Roel Baets ${ }^{1,2}$ (1)
}

\author{
${ }^{1}$ Photonics Research Group, Department of Information Technology, Ghent University-imec, Technologiepark-Zwijnaarde 15, 9052 Ghent, Belgium \\ ${ }^{2}$ Center for Nano- and Biophotonics (NB-Photonics), Ghent University, Technologiepark-Zwijnaarde 15, 9052 Ghent, Belgium \\ ${ }^{3}$ Faculty of Sciences, Department of Solid State Sciences, Ghent University, Krijgslaan 281/S1, 9000 Ghent, Belgium \\ ${ }^{4}$ Laboratoire d'Information Quantique, Université Libre de Bruxelles, Avenue Franklin D. Roosevelt 50, 1050 Brussels, Belgium \\ ${ }^{*}$ Corresponding author: artur.hermans@ugent.be
}

Received 21 December 2018; accepted 22 January 2019; posted 24 January 2019 (Doc. ID 355348); published 19 February 2019

\begin{abstract}
Silicon nitride ( $\mathrm{SiN})$ is currently the most prominent CMOS-compatible platform for photonics at wavelengths $<1 \mu \mathrm{m}$. However, realizing fast electro-optic (EO) modulators, the key components of any integrated optics platform, remains challenging in SiN. Modulators based on the plasma dispersion effect, as in silicon, are not available. Despite the fact that significant second-harmonic generation has been reported for silicon-rich SiN, no efficient Pockels effect-based modulators have been demonstrated. Here we report the back-end CMOS-compatible atomic layer deposition (ALD) of conventional second-order nonlinear crystals, zinc oxide, and zinc sulfide, on existing SiN waveguide circuits. Using these ALD overlays, we demonstrate EO modulation in ring resonators. (c) 2019 Optical Society of America
\end{abstract}

https://doi.org/10.1364/OL.44.001112

Today, silicon photonics is unmistakably one of the main photonic integration platforms. A key contributor to its success is the use of CMOS infrastructure for fabrication of photonic chips, enabling low-cost photonic integrated circuits (PICs), along with the possibility of electronic-photonic co-integration. Next to the established silicon-on-insulator (SOI) platform, plasmaenhanced chemical vapor deposition (PECVD) silicon nitride $(\mathrm{SiN})$ is gaining ground as a back-end CMOS-compatible material platform. Unlike silicon, SiN is transparent in the visible wavelength range [1]. Together with its low losses, it opens roads to new applications.

Electro-optic (EO) modulators are very useful components to have on a photonics platform. With the ever increasing demand for data, cheap, efficient, and fast optical modulators are in high demand. Next to telecom and datacom, other application areas are emerging where integrated $\mathrm{SiN}$ phase modulators can play an important role: programmable PICs for, e.g., microwave photonics and quantum information processing $[2,3]$, and optical phased arrays for, e.g., LIDAR and holographic 3D displays, requiring visible wavelength operation [4].

On the SOI platform, the plasma dispersion effect is exploited to realize fast $\mathrm{EO}$ modulators [5]. SiN however, is an insulating material, making the free charge carrier-based approach nonviable. Another well-known approach to $\mathrm{EO}$ modulation is the use of the Pockels effect. Lithium niobate $\left(\mathrm{LiNbO}_{3}\right)$ is particularly known for its large Pockels effect. Moreover, it is transparent in the visible and near-infrared wavelength range. Low-voltage integrated $\mathrm{LiNbO}_{3}$ modulators operating at $1550 \mathrm{~nm}$ have been demonstrated [6]. However, the use of thin film $\mathrm{LiNbO}_{3}$-oninsulator requires expensive bonding procedures. Unfortunately, the second-order nonlinearity of $\mathrm{SiN}$ and, consequently the Pockels effect, is expected to vanish due to SiN's amorphous nature. Yet, EO modulation has been demonstrated in low-loss LPCVD (low pressure CVD) SiN, albeit with small EO coefficients of maximum $8.31 \pm 5.6 \mathrm{fm} / \mathrm{V}$ at $1550 \mathrm{~nm}$ [7]. In addition, second-harmonic generation has been observed in SiN films [8] and waveguides [9]. Experiments indicate the existence of a bulk nonlinearity which appears to be strongest in siliconrich SiN films. It is yet unclear whether large Pockels coefficients in low-loss waveguides can be achieved.

As SiN itself offers no clear-cut solutions for optical modulation, apart from the slow and inefficient thermo-optic effect [10], there have been attempts to combine SiN with other materials. Combining graphene with $\mathrm{SiN}$, Phare et al. demonstrated a high-speed electro-absorption modulator at $1550 \mathrm{~nm}$ [11]. The fabrication was done by transferring CVD graphene on copper to a planarized SiN PIC. Mehta et al. showed efficient modulation in SiN-on- $\mathrm{LiNbO}_{3}$ Mach-Zehnder modulators at $674 \mathrm{~nm}$ [12]. Only DC characterization was performed. The modulators were fabricated by depositing PECVD SiN on a $\mathrm{LiNbO}_{3}$ substrate. Lead zirconate titanate (PZT) thin films have been used to demonstrate high-speed PZT-on-SiN modulators in the $\mathrm{O}$ - and C-band, with the possibility of extension to visible wavelengths [13]. However, the PZT deposition involves annealing at a temperature of $620^{\circ} \mathrm{C}$, which can cause $\mathrm{Cu}$ diffusion in the underlying CMOS electronics for back-end deposited photonic circuits [14]. In addition, AlN has been proposed as an alternative CMOS-compatible platform which is transparent at wavelengths $<1 \mu \mathrm{m}$ and allows for EO modulation [15].

In this Letter, we demonstrate monolithically integrated $\mathrm{SiN}$ based fast $\mathrm{EO}$ ring modulators operating at $0.9 \mu \mathrm{m}$, a wavelength 
often used in LIDAR systems [16]. The modulators are realized by overlaying $\mathrm{SiN}$ waveguides with atomic layer deposited (ALD) EO materials, namely zinc oxide $(\mathrm{ZnO})$ or zinc sulfide $(\mathrm{ZnS})$. ALD is a low-temperature conformal deposition technique compatible with CMOS fabrication technology [17]. Our approach does not require poling. Poled materials can suffer from long-term stability issues, especially at elevated operating temperatures. $\mathrm{ZnO}$ is a wide bandgap material $(\approx 3.4 \mathrm{eV})$ that crystallizes preferably in the hexagonal wurtzite structure, point group $6 \mathrm{~mm}$ [18]. $6 \mathrm{~mm}$ is a non-centrosymmetric point group, allowing for a Pockels effect. For bulk $\mathrm{ZnO}$ crystals, Pockels coefficients of $\left|r_{33}\right|=2.6 \mathrm{pm} / \mathrm{V}$ and $\left|r_{13}\right|=1.4 \mathrm{pm} / \mathrm{V}$ are reported at a wavelength of $0.63 \mu \mathrm{m}$ [19]. ZnS exists in two main non-centrosymmetric crystalline phases: the hexagonal wurtzite structure (bandgap $\approx 3.9 \mathrm{eV}$ ) and the cubic zinc-blende structure, point group $\overline{4} 3 \mathrm{~m}$ (bandgap $\approx 3.7 \mathrm{eV}$ ) [20]. At a wavelength of $0.63 \mu \mathrm{m}$, Pockels coefficients of $\left|r_{33}\right|=1.9 \mathrm{pm} / \mathrm{V}$ and $\left|r_{13}\right|=0.9 \mathrm{pm} / \mathrm{V}$ are reported for the wurtzite phase [19], and $\left|r_{41}\right|=1.9 \mathrm{pm} / \mathrm{V}$ for the zinc-blende phase [21]. To the best of our knowledge, no Pockels coefficients have been reported for these materials in thin films.

Our SiN waveguides are patterned in a CMOS pilot line using $193 \mathrm{~nm}$ deep UV lithography. The SiN layer has a thickness of $300 \mathrm{~nm}$ and is deposited by LPCVD on top of a $3.3 \mu \mathrm{m} \mathrm{SiO}{ }_{2}$ layer. On the patterned waveguides, the ALD materials are deposited (thicknesses in a 70-100 nm range) in a homebuilt setup with a base pressure of $10^{-6}$ mbar. A standard ALD cycle consists of a $5 \mathrm{~s}$ exposure for the precursor/co-reactants and a $25 \mathrm{~s}$ purge time between each exposure. The pressure of the precursor/co-reactants is set to $5 \times 10^{-3} \mathrm{mbar}$, unless stated otherwise. For the $\mathrm{ZnO}$ layers, two processes were used, both at a substrate temperature of $300^{\circ} \mathrm{C}$ : a plasma-enhanced ALD (PE-ALD) process using diethylzinc (DEZ) and $10 \mathrm{~s}$ oxygen plasma exposures $\left(10^{-2} \mathrm{mbar}\right)$, and a plasma-enhanced thermal ALD (PET-ALD) process using the exposure sequence DEZ- $\mathrm{H}_{2} \mathrm{O}-10 \mathrm{~s} \mathrm{O}_{2}$ plasma. The $\mathrm{O}_{2}$ plasma is generated by a radio frequency inductively coupled generator, operated at $13.56 \mathrm{MHz}$ and $200 \mathrm{~W}$ power. $\mathrm{ZnS}$ layers were deposited by a thermal process (THALD) using DEZ and $\mathrm{H}_{2} \mathrm{~S}$ at a substrate temperature of

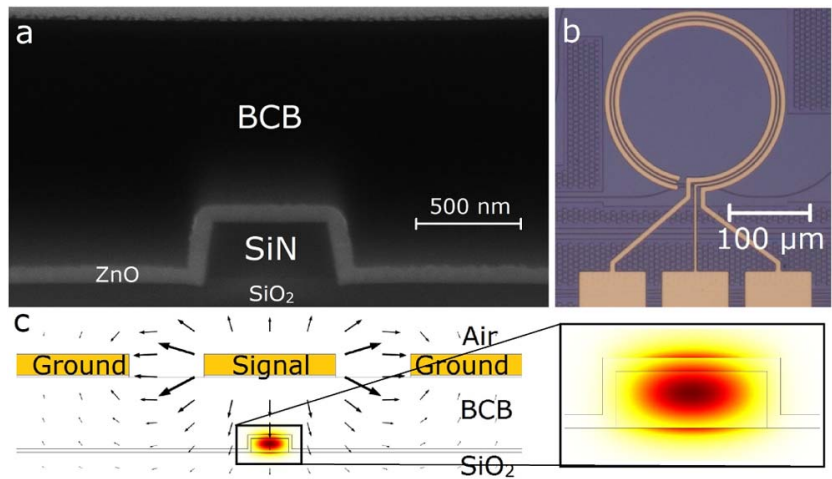

Fig. 1. (a) SEM image of the cross section of a $\mathrm{SiN}$ waveguide with $6 \mathrm{~nm} \mathrm{Al} \mathrm{O}_{3}, 81 \mathrm{~nm}$ PET-ALD $\mathrm{ZnO}$, and a $\mathrm{BCB}$ cladding. (b) Microscope image of a fabricated modulator. Rectangular contact pads are used for landing a GSG probe. (c) Schematic cross section of a $\mathrm{ZnO}$-covered $\mathrm{SiN}$ waveguide with electrodes. The intensity of the fundamental quasi-TM optical mode is shown, along with the electric field induced by the electrodes (arrows). 13\% of the mode power resides in the ALD overlay. $150^{\circ} \mathrm{C}$. In addition, the use of a $6 \mathrm{~nm} \mathrm{Al}_{2} \mathrm{O}_{3}$ seed layer (deposited prior to the $\mathrm{ZnO} / \mathrm{ZnS}$ layer) was investigated, as it has been shown to promote $\mathrm{ZnO}$ crystallite growth where the c-axis is perpendicular to the substrate $[22,23]$. This leads to an enhanced second-order nonlinearity. $\mathrm{Al}_{2} \mathrm{O}_{3}$ was deposited using trimethylaluminum and $\mathrm{H}_{2} \mathrm{O}$ at a substrate temperature of $120^{\circ} \mathrm{C}$. After $\mathrm{ALD}$, a layer of bisbenzocyclobutene (BCB) is spin coated and cured to serve as waveguide cladding. Figure 1(a) shows an SEM image of a waveguide cross section. Next, $\mathrm{Ti} / \mathrm{Au}$ ( $\mathrm{Ti}$ improves adhesion) electrodes are patterned in a ground-signal-ground (GSG) configuration utilizing a lift-off process. Figure 1(b) shows a fabricated device. Finally, the chips are cleaved to allow for edge coupling.

X-ray diffraction (XRD) $\theta-2 \theta$ scans were performed to check the crystallinity of our ALD thin films (Fig. 2). For $\mathrm{ZnO}$, the 002 reflection is clearly observed for all the tested ALD recipes, indicating that the crystallites preferentially grow with their c-axis normal to the substrate [22]. For the PET-ALD $\mathrm{ZnO}$, we can see that adding a thin $\mathrm{Al}_{2} \mathrm{O}_{3}$ layer increases the 002 reflection. For $\mathrm{ZnS}$, there are peaks at $28.2^{\circ}$ and $32.7^{\circ}$. The former can be ascribed to the 111 reflection of cubic $\mathrm{ZnS}$ [24], and the latter can be ascribed to the forbidden 200 reflection of the silicon substrate whose amplitude depends on the in-plane sample rotation [25]. The addition of $\mathrm{Al}_{2} \mathrm{O}_{3}$ slightly enhances the crystallinity. We expect textured films to be beneficial in preserving as much as possible of the single-crystal material nonlinearity.

Since our polycrystalline ALD films possess in-plane isotropy ( $\infty \mathrm{m}$ symmetry), the non-zero elements of their Pockels tensor are $r_{13}=r_{23}, r_{51}=r_{42}$, and $r_{33}$, with 3 referring to the normal to the film plane [15]. By choosing the electrode configuration illustrated in Fig. 1(c), we get an out-of-plane electric field in the film on the waveguide top surface, so we can probe $r_{13}$ (for TE modes) and $r_{33}$ (for TM modes). The effective index change of the guided mode depends on the EO coefficients, the overlap of the optical mode with the EO active layer, and the electric field induced by the electrodes [26]. In choosing the $\mathrm{BCB}$ cladding thickness, there is a trade-off between minimal absorption loss by the metal and maximum electric field in the thin film. We have $\mathrm{BCB}$ thicknesses of $\approx 1.6 \mu \mathrm{m}$ (measured from the top of the $\mathrm{SiO}_{2}$ layer) for the $\mathrm{ZnO}$-based devices and $\approx 1.5 \mu \mathrm{m}$ for the $\mathrm{ZnS}$-based devices. This gives an estimated (from mode simulations) absorption loss on the order of $0.001 \mathrm{~dB} / \mathrm{cm}$ for the fundamental quasi-TE mode and $0.01 \mathrm{~dB} / \mathrm{cm}$ for the quasi-TM mode, for a $\mathrm{SiN}$ waveguide width of $800 \mathrm{~nm}$ at a wavelength of $0.9 \mu \mathrm{m}$. The signal electrode width is chosen to be $2 \mu \mathrm{m}$ wider than the waveguide width to have some tolerance against misalignment in the
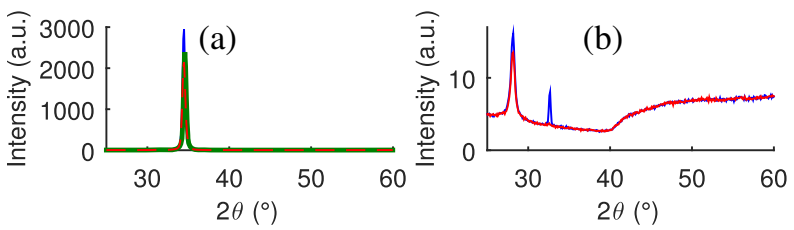

Fig. 2. XRD $\theta-2 \theta$ scans using $\mathrm{Cu} \mathrm{K} \alpha$ radiation for (a) $81 \mathrm{~nm}$ PETALD $\mathrm{ZnO}$ with $6 \mathrm{~nm} \mathrm{Al}_{2} \mathrm{O}_{3}$ seed layer (blue), $81 \mathrm{~nm}$ PET-ALD ZnO (red), $62 \mathrm{~nm}$ PE-ALD ZnO (green), and (b) $30 \mathrm{~nm}$ TH-ALD ZnS with $6 \mathrm{~nm} \mathrm{Al}{ }_{2} \mathrm{O}_{3}$ seed layer (blue), $27 \mathrm{~nm} \mathrm{TH-ALD} \mathrm{ZnS}$ (red). All films are deposited on silicon substrates with native oxide (thickness on the order of $1 \mathrm{~nm}$ ). 
Table 1. Propagation Loss for a SiN Waveguide Width of $800 \mathrm{~nm}$, for the Fundamental Quasi-TE and -TM Mode

\begin{tabular}{lcc} 
ALD Overlay & $\boldsymbol{\alpha}_{\mathrm{TE}}(\mathbf{d B} / \mathbf{c m})$ & $\boldsymbol{\alpha}_{\mathrm{TM}}(\mathbf{d B} / \mathbf{c m})$ \\
\hline No ALD overlay & $1.1 \pm 0.5$ & $0.9 \pm 0.2$ \\
PE-ALD ZnO & $11 \pm 4$ & $12 \pm 2$ \\
PET-ALD $\mathrm{ZnO}$ & $4 \pm 2$ & $3.9 \pm 0.6$ \\
PET-ALD $\mathrm{ZnO}+\mathrm{Al}_{2} \mathrm{O}_{3}$ & $4.6 \pm 0.7$ & $6 \pm 2$ \\
TH-ALD $\mathrm{ZnS}$ & $4 \pm 1$ & $9 \pm 2$ \\
TH-ALD $\mathrm{ZnS}+\mathrm{Al}_{2} \mathrm{O}_{3}$ & $3.0 \pm 0.8$ & $7.9 \pm 0.8$ \\
\hline
\end{tabular}

lithography process. The gap between the signal and ground electrodes is $1.6 \mu \mathrm{m}$. This gap size is limited by the resolution of our contact mask. Smaller gaps would result in larger electric fields, but can give issues during lift-off. In addition, smaller gaps increase the probability of dielectric breakdown. To convert the effective index change induced by the EO effect into a change in optical power, we use $100 \mu \mathrm{m}$ radius ring resonators. The coupling gaps between the ring and bus waveguides vary from 200 to $450 \mathrm{~nm}$ (excluding the ALD layer).

Both transmission and modulation measurements were conducted on the fabricated devices. In the transmission measurements, light from a tunable laser is coupled in and out of the chip via lensed fibers. The polarization is controlled with a fiber polarization controller. The resonances in the transmission spectrum can be fitted, which gives us the self-coupling coefficient $r$ and single-pass amplitude transmission $a$. Since various coupling gaps are available, $r$ and $a$ can be distinguished from one another, thus allowing us to get an estimate of the propagation loss [27]. Table 1 summarizes the estimated propagation losses for a SiN waveguide width of $800 \mathrm{~nm}$ at a wavelength of $0.9 \mu \mathrm{m}$. Figure 3(a) shows an example of a transmission measurement.

For the modulation measurements we apply a small AC voltage $U(t)=U_{\text {peak }} \sin (2 \pi f t)$ with a GSG probe. The transmitted light is led to a photodetector of which the $\mathrm{RF}$ output is connected to an electrical spectrum analyzer (ESA). On the ESA, a peak is observed at the same frequency as the applied voltage. From the ESA peak height, the variation in optical power $\Delta P_{\text {out }}$ can be calculated $\left(P_{\text {out }}(t)=\right.$ $\left.P_{\text {out }, 0}+\Delta P_{\text {out }} \sin (2 \pi f t)\right)$. Figure $3(\mathrm{a})$ shows how $\Delta P_{\text {out }}$ changes when the laser wavelength is tuned (for a fixed $U_{\text {peak }}$ and $f$ ). We see the maximum on the slopes of the res- (a)

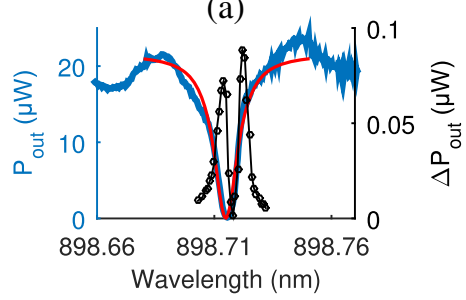

(b)

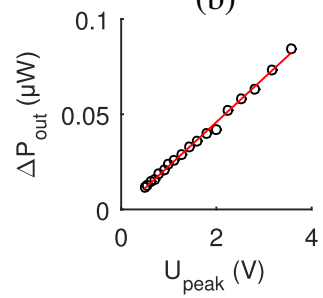

Fig. 3. Transmission and modulation measurements performed on a $\mathrm{SiN}$ with $\mathrm{ZnS}$ overlay (thickness of $100 \mathrm{~nm}$ ) ring modulator with a gap of $350 \mathrm{~nm}$ and a waveguide width of $800 \mathrm{~nm}$ for the quasi-TE mode. (a) Blue, transmitted optical power measured as a function of wavelength; red, fitting of resonance; black, modulation amplitude of transmitted optical power $\Delta P_{\text {out }}$ measured as a function of the wavelength for $f=10 \mathrm{MHz}$ and $U_{\text {peak }}=3.6 \mathrm{~V}$. (b) Black circles, measurement of $\Delta P_{\text {out }}$ as a function of $U_{\text {peak }}$ for $f=10 \mathrm{MHz}$ and a wavelength of $898.721 \mathrm{~nm}$. Red line, linear fitting of data. onance, while $\Delta P_{\text {out }}$ drops significantly at the bottom of the resonance and when tuning away from resonance. A slight shift in resonance occurred during the measurements. Figure 3(b) illustrates the linear variation of $\Delta P_{\text {out }}$ with the applied voltage $U_{\text {peak }}$. The aforementioned observations concur with a linear EO effect. The same behavior was seen for all the tested modulators. From the slope of the fitted line in Fig. 3(b) and the first-order derivative of the transmission spectrum at the bias point, the $V_{\pi} L$ product can be estimated:

$$
V_{\pi} L \approx \frac{\lambda^{2} L}{n_{\mathrm{eff}} 4 \pi R} \frac{\frac{\partial P_{\text {out }}}{\partial \lambda}}{\frac{\Delta P_{\text {out }}}{U_{\text {peak }}}},
$$

with $\lambda$ being the wavelength, $L$ being the electrode length, $n_{\text {eff }}$ being the effective refractive index of the optical mode, and $R$ being the ring radius. For the $\mathrm{ZnO}$-based devices, we got the following $V_{\pi} L$ estimates for the quasi-TE (TM) mode: 6(4) $\mathrm{V} \cdot \mathrm{m}$ for PE-ALD, $1 \times 10(2 \times 10) \mathrm{V} \cdot \mathrm{m}$ for PET-ALD, and $6(5) \mathrm{V} \cdot \mathrm{m}$ for PET-ALD with $\mathrm{Al}_{2} \mathrm{O}_{3}$. For the $\mathrm{ZnS}$ based devices, we found for the quasi-TE (TM) mode $2 \times 10(3 \times 10) \mathrm{V} \cdot \mathrm{m}$ without $\mathrm{Al}_{2} \mathrm{O}_{3}$ and $3 \times 10(2 \times 10) \mathrm{V} \cdot \mathrm{m}$ with $\mathrm{Al}_{2} \mathrm{O}_{3}$. For $\mathrm{SiN}$ modulators without an ALD overlay, we estimated $1 \times 10^{2}\left(2 \times 10^{2}\right) \mathrm{V} \cdot \mathrm{m}$ for the quasi-TE (TM) mode. For the PET-ALD $\mathrm{ZnO}$ overlay, the addition of $\mathrm{Al}_{2} \mathrm{O}_{3}$ improves $V_{\pi} L$, which can be related to the improved crystallinity. For the $\mathrm{ZnS}$ overlay, no significant improvement was observed. The PE-ALD $\mathrm{ZnO}$ overlay gives $V_{\pi} L$ values similar to the PET-ALD $\mathrm{ZnO}$ with $\mathrm{Al}_{2} \mathrm{O}_{3}$ overlay, but the loss is larger for the PE-ALD $\mathrm{ZnO}$ overlay.

In addition, the $\mathrm{EO}$ response was measured for increasing modulation frequency (Fig. 4). As the Pockels effect is an ultrafast effect, the bandwidth is expected to be limited by the cavity photon lifetime $\tau$ and/or the resistance-capacitance time constant. The $3 \mathrm{~dB}$ bandwidth can be estimated from $1 / f_{3 \mathrm{~dB}}^{2}=1 / f_{Q}^{2}+1 / f_{R C}^{2}=(2 \pi \tau)^{2}+(\pi(50 \Omega+2 R) C)^{2}$, where $50 \Omega$ is the output impedance of the signal generator and termination of the probe; $R$ and $C$ are the series resistance and capacitance of the device [5]. From $S_{11}$ parameter measurements, we found $C=140 \mathrm{fF}$ and $R=200 \Omega$. For the $\mathrm{ZnS}$-based modulators and blank SiN modulator measured in Fig. 4(b), we estimated the photon lifetimes $\tau$ and corresponding $f_{3 \mathrm{~dB}}: 29 \mathrm{ps}$, corresponding to $3.7 \mathrm{GHz}$ (red), 37 ps, corresponding to $3.3 \mathrm{GHz}$ (blue), and $97 \mathrm{ps}$, corresponding to $1.5 \mathrm{GHz}$ (black). The observed bandwidths are somewhat smaller; however, this is not unusual, as the above-mentioned empirical formula does not account for coupling conditions and wavelength [5]. For the $\mathrm{ZnO}$-based modulators, none of the above explains the $\approx 100 \mathrm{MHz}$
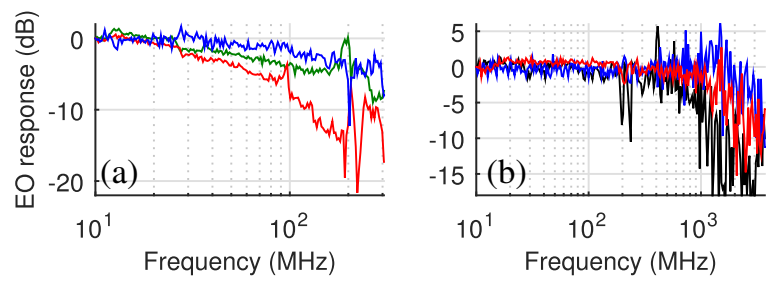

Fig. 4. EO response of modulators versus modulation frequency. (a) PE-ALD $\mathrm{ZnO}$ (green), PET-ALD $\mathrm{ZnO}$ (red), and PET-ALD $\mathrm{ZnO}$ with $\mathrm{Al}_{2} \mathrm{O}_{3}$ (blue). (b) TH-ALD $\mathrm{ZnS}$ (red), TH-ALD ZnS with $\mathrm{Al}_{2} \mathrm{O}_{3}$ (blue), and $\mathrm{SiN}$ without ALD overlay (black). 
bandwidths. $\mathrm{ZnO}$ is known to possess n-type conductivity due to the presence of defects and impurities [28]. The free charge carriers could play a role in the modulation mechanism. The resistivity $\rho$ of the ALD films was measured with a four-point probe. For the PE-ALD ZnO films, we found $\rho \approx 10 \Omega \cdot \mathrm{cm}$. For all other $\mathrm{ZnO}$ and $\mathrm{ZnS}$ films, $\rho$ was above the measurement limit $\left(>10^{4} \Omega \cdot \mathrm{cm}\right)$. The PET-ALD recipe was used specifically to increase $\rho$ and decrease the carrier concentration [29]. Although $\rho$ was increased by several orders of magnitude, this had no significant influence on the bandwidth. To investigate the role of free carriers in the modulation mechanism and extend the bandwidth, a more in-depth material exploration is required, including annealing treatments and depositions at different temperatures to alter the film's resistivity [28].

From the knowledge of $V_{\pi} L$, the simulated electrostatic and optical field distributions, the Pockels coefficients can be estimated [26]. For SiN, we estimate $\left|r_{33}\right| \approx 0.01 \mathrm{pm} / \mathrm{V}$ and $\left|r_{13}\right| \approx 0.02 \mathrm{pm} / \mathrm{V}$. For $\mathrm{ZnS}$ (with $\mathrm{Al}_{2} \mathrm{O}_{3}$ ), we find $\left|r_{33}\right| \approx$ $0.3 \mathrm{pm} / \mathrm{V}(0.4 \mathrm{pm} / \mathrm{V})$ and $\left|r_{13}\right| \approx 0.2 \mathrm{pm} / \mathrm{V}(0.1 \mathrm{pm} / \mathrm{V})$. These values are smaller than those reported for bulk $\mathrm{ZnS}$ crystals, as expected for polycrystalline films. For PE-ALD $\mathrm{ZnO}$, we estimate $\left|r_{33}\right| \approx 3 \mathrm{pm} / \mathrm{V}$ and $\left|r_{13}\right| \approx 2 \mathrm{pm} / \mathrm{V}$. For PETALD $\mathrm{ZnO}$ (with $\mathrm{Al}_{2} \mathrm{O}_{3}$ ), we find $\left|r_{33}\right| \approx 0.5 \mathrm{pm} / \mathrm{V}(2 \mathrm{pm} / \mathrm{V})$ and $\left|r_{13}\right| \approx 0.7 \mathrm{pm} / \mathrm{V}(2 \mathrm{pm} / \mathrm{V})$. Some of these values are larger than those reported for bulk $\mathrm{ZnO}$ crystals, which also hints to the fact that other mechanisms play a role in the refractive index modulation.

Although the estimated $V_{\pi} L$ values are large, we do not need a full $\pi$ phase shift in our ring resonators to realize switching or modulation with a large extinction ratio. For instance, for a critically coupled ring with a loss of $5 \mathrm{~dB} / \mathrm{cm}$, switching between the minimum transmission ( 0 at critical coupling) and a transmission of $-3 \mathrm{~dB}$ (not including coupling and access waveguide loss) requires only $2 \%$ of $V_{\pi}$.

By adapting the waveguide dimensions and electrode configuration, further improvements in $V_{\pi} L$ can be made. First, the modal overlap with the EO ALD layer can be increased by reducing the SiN thickness and/or increasing the ALD layer thickness. Secondly, the electric field strength can be increased by lowering the ground electrodes (placing them in trenches etched in the $\mathrm{SiO}_{2}$ next to the waveguide). This can reduce the $V_{\pi} L$ product by at least a factor of 5 . Other measures to increase the field strength are the use of transparent conducting oxides as electrodes which can be placed very close to the waveguide core [30], or using of nanoplasmonic SiN slot waveguides [31].

In conclusion, we demonstrated fast SiN-based EO ring modulators by overlaying SiN PICs with an EO material, namely $\mathrm{ZnO}$ or $\mathrm{ZnS}$. These materials are deposited by ALD, a lowtemperature deposition technique compatible with CMOS fabrication technology. Considering the lack of CMOS-compatible SiN-based EO modulators, our results are very valuable.

Funding. Fonds Wetenschappelijk Onderzoek (FWO); Fonds De La Recherche Scientifique-FNRS (FNRS).

\section{REFERENCES}

1. A. Rahim, E. Ryckeboer, A. Z. Subramanian, S. Clemmen, B. Kuyken, A. Dhakal, A. Raza, A. Hermans, M. Muneeb, S. Dhoore, Y. Li, U. Dave, P. Bienstman, N. Le Thomas, G. Roelkens, D. Van
Thourhout, P. Helin, S. Severi, X. Rottenberg, and R. Baets, J. Lightwave Technol. 35, 639 (2017).

2. L. Zhuang, C. G. H. Roeloffzen, M. Hoekman, K.-J. Boller, and A. J. Lowery, Optica 2, 854 (2015).

3. C. Taballione, T. A. W. Wolterink, J. Lugani, A. Eckstein, B. A. Bell, R. Grootjans, I. Visscher, D. Geskus, C. G. H. Roeloffzen, J. J. Renema, I. A. Walmsley, P. W. H. Pinkse, and K.-J. Boller, " $8 \times 8$ programmable quantum photonic processor based on silicon nitride waveguides," arXiv:1805.10999 (2018).

4. M. Raval, A. Yaacobi, D. Coleman, N. M. Fahrenkopf, C. Baiocco, G. Leake, T. N. Adam, D. Coolbaugh, and M. R. Watts, in IEEE Photonics Conference (2016), paper MG3.4.

5. H. Yu, D. Ying, M. Pantouvaki, J. Van Campenhout, P. Absil, Y. Hao, J. Yang, and X. Jiang, Opt. Express 22, 15178 (2014).

6. C. Wang, M. Zhang, X. Chen, M. Bertrand, A. Shams-Ansari, S. Chandrasekhar, P. Winzer, and M. Lončar, Nature 562, 101 (2018).

7. S. Miller, Y.-H. D. Lee, J. Cardenas, A. L. Gaeta, and M. Lipson, in Conference on Lasers and Electro-Optics (2015), paper SF1G.4.

8. E. F. Pecora, A. Capretti, G. Miano, and L. Dal Negro, Appl. Phys. Lett. 102, 141114 (2013).

9. M. W. Puckett, R. Sharma, H.-H. Lin, M.-H. Yang, F. Vallini, and Y. Fainman, Opt. Express 24, 16923 (2016).

10. J. Joo, J. Park, and G. Kim, IEEE Photonics Technol. Lett. 30, 740 (2018).

11. C. T. Phare, Y.-H. D. Lee, J. Cardenas, and M. Lipson, Nat. Photonics 9, 511 (2015).

12. K. K. Mehta, G. N. West, and R. J. Ram, in Conference on Lasers and Electro-Optics (2017), paper STu3N.7.

13. K. Alexander, J. P. George, J. Verbist, K. Neyts, B. Kuyken, D. Van Thourhout, and J. Beeckman, Nat. Commun. 9, 3444 (2018).

14. Y. H. D. Lee and M. Lipson, IEEE J. Sel. Top. Quantum Electron. 19, 8200207 (2013).

15. C. Xiong, W. H. P. Pernice, and H. X. Tang, Nano Lett. 12, 3562 (2012).

16. C. V. Poulton, A. Yaacobi, D. B. Cole, M. J. Byrd, M. Raval, D. Vermeulen, and M. R. Watts, Opt. Lett. 42, 4091 (2017).

17. K. Mistry, C. Allen, C. Auth, B. Beattie, D. Bergstrom, M. Bost, M. Brazier, M. Buehler, A. Cappellani, R. Chau, C.-H. Choi, G. Ding, K. Fischer, T. Ghani, R. Grover, W. Han, D. Hanken, M. Hattendorf, J. He, J. Hicks, R. Huesnner, D. Ingerly, P. Jain, R. James, L. Jong, S. Joshi, C. Kenyon, K. Kuhn, K. Lee, H. Liu, J. Maiz, B. Mclntyre, P. Moon, J. Neirynck, S. Pae, C. Parker, D. Parsons, C. Prasad, L. Pipes, M. Prince, P. Ranade, T. Reynolds, J. Sandford, L. Shifren, J. Sebastian, J. Seiple, D. Simon, S. Sivakumar, P. Smith, C. Thomas, T. Troeger, P. Vandervoorn, S. Williams, and K. Zawadzki, in IEEE International Electron Devices Meeting (2007), pp. 247-250.

18. M. C. Larciprete and M. Centini, Appl. Phys. Rev. 2, 031302 (2015).

19. I. P. Kaminov and E. H. Turner, Proc. IEEE 54, 1374 (1966).

20. S. Kasap and P. Capper, Springer Handbook of Electronics and Photonic Materials, 1st ed. (Springer, 2007).

21. L. Baldassarre, A. Cingolani, M. Ferrara, and M. Lugarà, Solid State Commun. 34, 237 (1980).

22. A. Wickberg, C. Kieninger, C. Sürgers, S. Schlabach, X. Mu, C. Koos, and M. Wegener, Adv. Opt. Mater. 4, 1203 (2016).

23. A. Hermans, M. Van Daele, C. Kieninger, J. Dendooven, S. Clemmen, C. Detavernier, C. Koos, and R. Baets, in Conference on Lasers and Electro-Optics (2017), paper SM3K.3.

24. J. Kuhs, T. Dobbelaere, Z. Hens, and C. Detavernier, J. Vac. Sci. Technol. A 35, 01B111 (2017).

25. P. Zaumseil, J. Appl. Crystallogr. 48, 528 (2015).

26. C. Koos, "Nanophotonic devices for linear and nonlinear optical signal processing," Ph.D. dissertation (Universität Karlsruhe [TH], 2007).

27. W. Bogaerts, P. De Heyn, T. Van Vaerenbergh, K. De Vos, S. K. Selvaraja, T. Claes, P. Dumon, P. Bienstman, D. Van Thourhout, and R. Baets, Laser Photonics Rev. 6, 47 (2012).

28. T. Tynell and M. Karppinen, Semicond. Sci. Technol. 29, 043001 (2014).

29. M. A. Thomas and J. B. Cui, ACS Appl. Mater. Interfaces 4, 3122 (2012).

30. G. Xu, Z. Liu, J. Ma, B. Liu, S.-T. Ho, L. Wang, P. Zhu, T. J. Marks, J. Luo, and A. K. Y. Jen, Opt. Express 13, 7380 (2005).

31. A. Raza, S. Clemmen, P. Wuytens, M. Muneeb, M. Van Daele, J. Dendooven, C. Detavernier, A. Skirtach, and R. Baets, APL Photonics 3, 116105 (2018). 